\title{
A COMPARISON OF THE SAFETY AND EFFICACY OF THE VENABLOCK CYANOACRYLATE-BASED ENDOVENOUS SYSTEM VERSUS 1470 NM ENDOVASCULAR BIRADIAL LASER IN THE TREATMENT OF TRUNCAL INSUFFICIENCY OF SUPERFICIAL VEINS: SIX-MONTH OUTCOMES OF THE ESVETIS OBSERVATIONAL STUDY
}

\author{
Justyna Wilczko', Cezary Szary¹, Dominika Plucińska', Tomasz Grzela ${ }^{1,2}$ \\ 'Phlebology Clinic, Warsaw, Poland \\ 2Department of Histology and Embryology, Medical University of Warsaw, Warsaw, Poland
}

\begin{abstract}
Introduction: Current treatment of superficial vein insufficiency is based on minimally invasive methods, among them the VenaBlock system, a next-generation cyanoacrylate glue. The modified glue formula results in low viscosity and fast polymerisation, which may affect the accuracy and effectiveness of the treatment. The aim of our observational study was to assess the safety and efficacy of the VenaBlock system and compare it to near-infrared $(1470 \mathrm{~nm})$ laser thermoablation.

Material and methods: The study involved 87 patients allocated to either VenaBlock $(n=41)$ or laser $(n=46)$ groups. The assessment comprised selected morphometric parameters, duration of procedure, pain during and after procedure, occurrence of adverse events, changes in patients' quality of life score, and effectiveness of vein closure during six-month follow-up.

Results: The VenaBlock procedure was shorter in comparison with laser treatment (7.1 \pm 4.6 vs. $17.0 \pm 4.2$ minutes, respectively); pain during the procedure was more severe in the VenaBlock than in the laser group ( $3.5 \pm 2.3$ vs. $2.7 \pm 2.0$, respectively), but this difference was not statistically significant. However, one week after the treatment, pain was significantly more severe in the VenaBlock group $(4.3 \pm 2.4$ vs. 2.9 \pm 2.4 , respectively). Neither serious adverse events nor other significant adverse reactions were observed. The six-month follow-up revealed a $90.3 \%$ occlusion rate in the VenaBlock group vs. $97.8 \%$ in the laser group; this difference was not statistically significant. However, the use of VenaBlock in larger veins was associated with higher risk of recanalisation.

Conclusions: The VenaBlock system is a safe and fast procedure, but its efficacy is limited in veins of large diameter.
\end{abstract}

Key words: endovascular treatment, cyanoacrylate glue, laser thermoablation, superficial vein.
ORIGINAL PAPER

Phlebological Review 2019; 27, 1: 3-9

DOI: https://doi.org/10.5114/pr.2019.93402

Submitted: 15.05 .2019

Accepted: 11.06 .2019

ADDRESS FOR CORRESPONDENCE
Tomasz Grzela
Department of Histology and Embryology
Medical University of Warsaw
5 Chałubińskiego St.
02-004 Warsaw, Poland
e-mail: tomekgrzela@gmail.com

ADDRESS FOR CORRESPONDENCE

Department of Histology and Embryology

5 Chałubińskiego St.

e-mail: tomekgrzela@gmail.com

\section{INTRODUCTION}

Current treatment of truncal incompetence of the superficial veins is based on minimally invasive endovascular methods, which comprise thermal and non-thermal (or chemical) ablation procedures [1]. Thermal techniques include radiofrequency, near-infrared laser, and super-heated steam-mediated ablations. Non-thermal methods comprise foam sclerotherapy and liquid sclerotherapy, mechano-chemical ablation, and a novel technique based on endovascular application of cyanoacrylate glue $[2,3]$. Few clinical trials provided promising data concerning the clinical usefulness of endovenous glue systems in the treatment of superficial vein incompetence [4-6]. The aforementioned reports, although very enthusiastic regarding the efficacy of glue and demonstrating non-inferiority of glue application in comparison with other methods, were based on short-term observations, and therefore they did not solve all issues and doubts, which require further studies [7].

Most recently, the VenaBlock system, a next-generation cyanoacrylate glue, was introduced to the market by the Turkish company Invamed Saglic Ilac A.S. It is based on low-viscosity/liquid glue, characterised by rapid (few 
seconds) polymerisation. The "all-you-need" kit of the VenaBlock system consists of a vial of cyanoacrylate solution, calibrated gun dispenser and a wire-reinforced catheter with a red diode-lighted tip, which allows easy localisation of its current position.

The modified glue formula and very fast polymerisation requires important changes in the application protocol compared to the VenaSeal system $[5,7]$. These changes include a larger volume of glue for a single application and faster pull-back of the catheter, which results in significant shortening of the procedure. However, such a modification may affect the accuracy and effectiveness of this method. Therefore, the aim of our prospective observational study was to assess the safety and efficacy of the VenaBlock system and compare it to the endovascular thermoablation, with the use of a near-infrared $(1470 \mathrm{~nm})$ laser with radial fibre. In this paper we show our preliminary results after one and six months.

\section{MATERIAL AND METHODS}

The study involved 87 patients, 59 females and 28 males (mean age $44.2 \pm 12.8$ years), with truncal incompetence of superficial veins ( $\mathrm{C} 2$ to $\mathrm{C} 4$ according to the CEAP classification), without involvement of deep veins. Inclusion and exclusion criteria are shown in Table 1. Incompetence of the great saphenous vein $(n=72)$, the small saphenous vein $(n=9)$, or the anterior accessory saphenous vein $(n=6)$ was confirmed by duplex-Doppler ultrasound examination, which was performed in the standing position. We applied the Xario 100 diagnostic ultrasound system with an $18 \mathrm{MHz}$ linear probe (TOSHIBA/Canon Medical Systems Co., Otawara, Tochigi, Japan). The morphometric assessment included: the length of incompetent trunk, the vein diameter at the level of the terminal valve (ter) in the sapheno-femoral (SFJ) or the sapheno-popliteal (SPJ) junctions, the diameter of the target vein at the level of the pre-terminal valve (pter), $5 \mathrm{~cm}$ below the pre-terminal valve (5pter), and at the level of the distal reflux point (dist) of the incompetent venous trunk.

Patients were assigned to two study arms: the laser (L) group or the VenaBlock (VB) group. All patients gave their informed consent to participate in the study.
The protocol of the ESVETIS (Efficacy and Safety of VenaBlock vs. ELVeS systems in the treatment of Truncal Insufficiency in Superficial veins of lower limbs) study was reviewed and approved by the Local Ethical Committee (approval No. KB/1073/17).

Before the treatment, patients were asked to selfassess their quality of life (QoL) using the questionnaire with eight single-choice questions, focused on selected venous insufficiency-related symptoms, including visible varicose veins, pain, leg oedema, and/or heaviness, etc. Each answer was rated on a four-point scale, where " 0 " corresponded to absence of the symptom, and " 4 " described a constant presence and significant severity of such a symptom. Thus, the minimal score (0) represented the best patient's wellbeing, without any symptom of the disease, while the maximal score (32) corresponded to an overall poor condition of patient's veins and high severity of venous symptoms.

\section{Laser group}

Patients allocated to the L group $(n=46)$ were treated using a $15 \mathrm{~W}$ near-infrared $(1470 \mathrm{~nm})$ diode laser (Leonardo Dual 45, Biolitec/CeramOptec GmbH, Bonn, Germany). A $1.8 \mathrm{~mm}$-diameter radial optic fibre (ELVeS Radial 2ring Fibre, CeramOptec GmbH, Bonn, Germany) was introduced under ultrasound guidance into the trunk of the target vein at the distal point of the axial reflux using a 6-F introducer (Prelude Sheath Introducer, Merit Medical Systems Inc., South Jordan, UT). The fibre tip was placed $3 \mathrm{~cm}$ below the sapheno-femoral or sapheno-popliteal junction. Then, tumescent anaesthesia with Klein's solution [8] was applied along the treated vein, using a peristaltic pump (Dispenser DP30, NOUVAG AG, Goldach, Switzerland). Ablation of the vein was performed using manual fibre traction, applying a linear endovenous energy density (LEED), which was calculated according to the formula: vein diameter in $\mathrm{mm}$ $\times 7=$ energy applied on each $\mathrm{cm}$ of the treated vein.

The duration of the procedure was measured from the time of venous access placement to the removal of the laser fibre. The volume of tumescent solution and laser energy applied were also recorded.

Table 1. Inclusion and exclusion criteria to enrol the patient

\begin{tabular}{ll}
\multicolumn{1}{c}{ Inclusion } & \multicolumn{1}{c}{ Exclusion } \\
\hline - $>18$ years & - Acute or sub-acute thrombosis (deep or superficial) \\
- Reflux $>1 \mathrm{sec}$ in ultrasound scan of GSV, AASV, or SSV trunk & - Significant insufficiency of deep veins \\
- CEAP - C2-C4 & - Previous interventions in treated vessel \\
- Initial diameter of incompetent trunk $>4 \mathrm{~mm},<10 \mathrm{~mm}$, as measured & - Pregnancy or breastfeeding \\
in standing position, $5 \mathrm{~cm}$ below (distally) of preterminal valve (5pter) & - Known allergy to cyanoacrylates \\
- The length of incompetent trunk $>20 \mathrm{~cm}$ & - Any severe or unstable (uncontrolled) chronic disease \\
& - Active cancer or any oncologic treatment within last 5 years
\end{tabular}


A small sterile dressing was placed on the site of the vein puncture after fibre removal, and a compression stocking was worn on the leg. Patients were advised to wear continuously compression stockings for two days after the procedure, and over next two weeks to use such a compression only during the day.

\section{VenaBlock group}

In patients allocated to the VB group $(n=41)$ the same 6-F introduction kit was used as in the L group. Local anaesthesia with $1 \mathrm{ml}$ of $1 \%$ lidocaine was applied only at the site of vein puncture. The wire-reinforced catheter with the red diode-lighted tip was connected to the syringe containing $2.0 \mathrm{ml}$ of cyanoacrylate and was mounted in the gun dispenser (all components included in VenaBlock Vein Sealing System, Invamed Saglic Ilac A.S., Ankara, Turkey). The prepared catheter was introduced to the target vein through the access port and its tip was placed under sonographic guidance $3 \mathrm{~cm}$ below the sapheno-femoral or sapheno-popliteal junction. Glue application $(0.3 \mathrm{ml}$ per each $10 \mathrm{~cm}$ of target vein) was performed with steady traction of the catheter, with simultaneous compression along the vein for 30 seconds, according to recommendations of the manufacturer.

Immediately after catheter removal the site of vein access was secured with a sterile dressing and the patient was mobilised. No compression stocking was applied to the leg. The volume of glue applied to the vein and the duration of the procedure, measured from the time of venous access placement to the removal of catheter, were recorded.

\section{Further treatment}

In both groups, at the end of procedure all patients received prophylactic doses of enoxaparin (Clexane, Sanofi-Aventis/CHINOIN Pharmaceutical and Chemical Works Private Co. Ltd, Miskolc, Hungary), which was administered for seven days. Immediately after the procedure patients were encouraged to walk for 30 minutes. Also, they were asked to estimate pain/discomfort during the entire procedure, using the 10-point visual analogue scale (VAS). If necessary, patients were allowed to use a non-steroidal anti-inflammatory drug (such as nimesulide, orally $100 \mathrm{mg}$ twice a day). Follow-up visits were scheduled for one and six months after the procedure. During all follow-up visits the general condition of patients, occurrence of adverse events, and any changes in medications were recorded. Furthermore, patients were asked to fill in QoL questionnaires. Efficacy of vein occlusion and diameter of the vein were measured at the same levels as before the treatment. Additionally, at onemonth follow-up, patients were asked to evaluate pain in the treated leg during the first week after the procedure using the 10-point VAS scoring system.

\section{Statistical analysis}

Data analysis was conducted using the BrightStat free online software (BrightStat.com). Comparative assessment of selected parameters between both tested groups was performed with the Mann-Whitney $U$-test, whereas changes within each group were analysed using the Wilcoxon-matched pairs test. The significance of the $p$ values was set at $p<0.05$. The odds ratios (OR) estimating a chance to achieve particular goal in assessed treatment regimen were calculated in regard to selected parameters with $95 \%$ confidence intervals (CI).

\section{RESULTS}

There were no statistically significant differences between study groups regarding patients' mean age, body mass index, and sex distribution. Also, the mean diameters in selected control points of target veins and mean QoL scores in pre-treatment assessment were similar in both study groups (Table 2). A significant difference was observed in duration of procedures. Except for four cases with some technical difficulties, resulting mainly from a strong and painful vein spasm during catheter introduction, in the VB group the time necessary for entire intervention did not exceed 10 minutes (mean $7.1 \pm 4.6$ minutes). In contrast, in the $\mathrm{L}$ group the mean time required for the procedure was $17.0 \pm 4.2$ minutes, and the shortest procedure did not last less than 9 minutes (Fig. 1). These differences were statistically significant $(p<0.001)$. The OR assessed for procedure duration longer than $10 \mathrm{~min}-$ utes in the VB group was 0.1 , with $95 \% \mathrm{CI}=0.03-0.3$ and $p=0.0001$ (Fig. 2).

Table 2. Clinical characteristics of patients from VenaBlock (VB) and laser (L) study groups

\begin{tabular}{lcc}
\hline Feature/parameter & \multicolumn{1}{c}{$\begin{array}{c}\text { VB group } \\
(\boldsymbol{n}=\mathbf{4 1 )}\end{array}$} & $\begin{array}{c}\text { L group } \\
(\boldsymbol{n}=\mathbf{4 6})\end{array}$ \\
\hline Mean (median) age & $46.1 \pm 14.4(41.5)$ & $42.5 \pm 11.2(41.0)$ \\
\hline $\begin{array}{l}\text { Sex distribution - } \\
\text { male/female (\%) }\end{array}$ & $13 / 28(31.7 / 68.3)$ & $15 / 31(32.6 / 67.4)$ \\
\hline Mean (median) BMl & $24.2 \pm 3.6(23.5)$ & $23.9 \pm 3.5(23.2)$ \\
\hline \begin{tabular}{l} 
Mean (median) initial vein diameter in mm, at the level of \\
\hline Terminal valve (ter)
\end{tabular} & $7.3 \pm 1.8(7.6)$ & $7.6 \pm 1.5(7.5)$ \\
\hline $\begin{array}{l}\text { Preterminal valve (pter) } \\
7.5 \pm 1.7(7.6)\end{array}$ & $8.2 \pm 1.7(8.0)$ \\
\hline $\begin{array}{l}5 \text { cm below preterminal } \\
\text { valve (5pter) }\end{array}$ & $5.3 \pm 1.2(4.8)$ & $5.4 \pm 1.4(5.1)$ \\
\hline $\begin{array}{l}\text { Mean (median) length of } \\
\text { treated vein in cm }\end{array}$ & $44.5 \pm 11.2(47.0)$ & $42.0 \pm 10.4(42.0)$ \\
\hline $\begin{array}{l}\text { Mean (median) initial quality } \\
\text { of life score }\end{array}$ & $13.9 \pm 6.4(14.0)$ & $14.9 \pm 6.2(16.0)$ \\
\hline
\end{tabular}




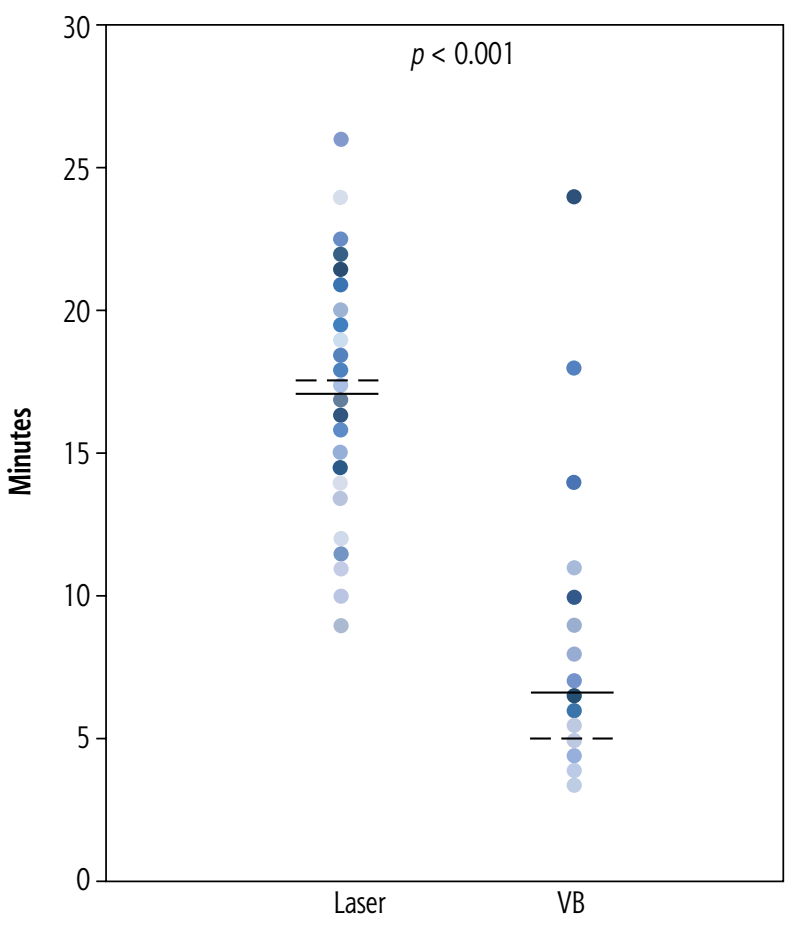

Fig. 1. Duration of procedure (in minutes) in VenaBlock (VB) and laser groups. Each dot represents one patient/procedure. Solid lines correspond to mean, whereas dashed lines correspond to median values

\section{Pain and quality of life}

Mean intra-operative pain/discomfort, assessed using the 10-point VAS, was more severe in the VenaBlock in comparison with the $\mathrm{L}$ group $(3.5 \pm 2.3$ vs. $2.7 \pm 2.0$, respectively). However, this difference was not statistically significant. OR assessed for level of intra-operative pain higher than five points during the procedure in the VB group was 1.8 with $95 \% \mathrm{CI}=0.47-6.89 ; p=0.39$ (Fig. 2). Assessment of postoperative pain, concerning seven days after the procedure, revealed significantly higher scores in the VB group. Pain was especially severe in six out of 41 patients $(14.6 \%)$. The pain probably resulted from the inflammatory reaction along the occluded vein, which was present despite the use of non-steroidal anti-inflammatory agents. Conversely, patients from the $\mathrm{L}$ group did not require postoperative pain management. Mean pain scores were $4.3 \pm 2.4$ vs. $2.9 \pm 2.4$, respectively $(p=0.006)$. The OR assessed for pain scores higher than five points during first post-procedural week in the VB group was 3.39, with $95 \% \mathrm{CI}=0.97-11.81 ; p=0.05$ (Fig. 2). Of note, the observed postoperative pain/discomfort and the differences between both groups were transient and disappeared completely within 10-12 days after the procedure. Pain was no longer reported during first follow-up, which was done one month after the procedure.

Postoperative assessment of QoL revealed significant decreases of individual scores in all treated patients in both groups. Comparison of mean QoL scores did not show any significant differences between the study groups one and six months after the procedure. The OR assessed for QoL decrease within six months after the procedure in the VB group was 1.33, with $95 \% \mathrm{CI}=0.46-3.86$; $p=0.59$ (Fig. 2). The frequencies of other adverse events, such as ecchymoses, bruising, discoloration, and paraesthesia, did not differ between study groups.

In both groups there were no serious adverse events during the procedure and during six-month follow-up.

\section{Morphology and efficacy}

In all patients from both groups after the procedure ultrasound examination of treated veins revealed significant reduction of their diameter in all specified control

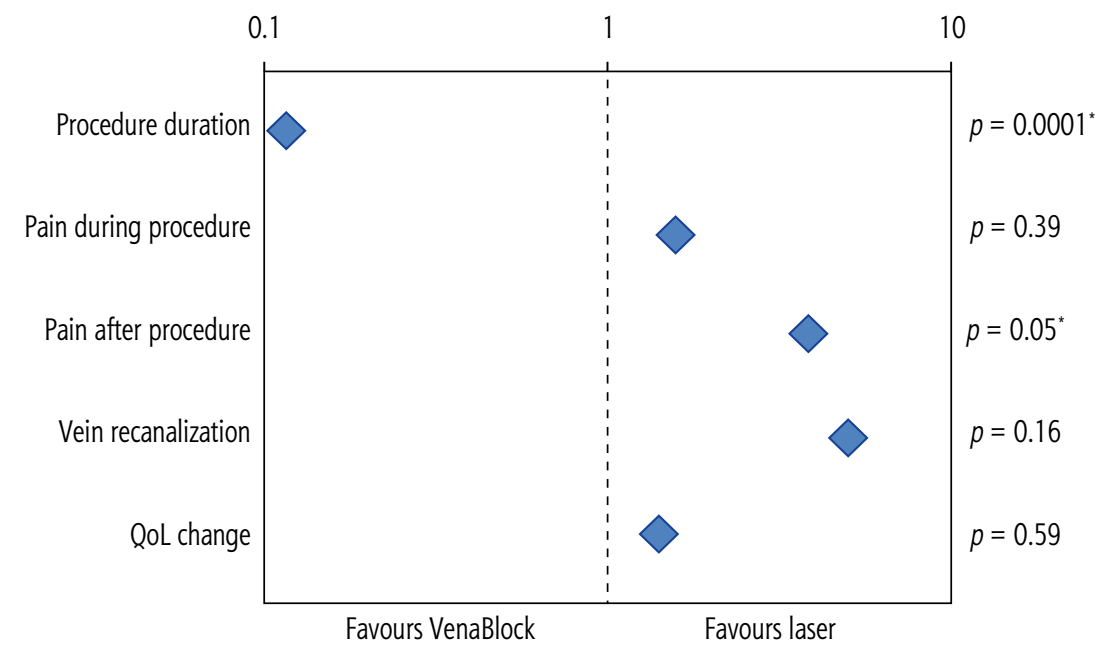

Fig. 2. Comparison of main features of both assessed methods. The odds ratios below 1.0 will favour VenaBlock, whereas above 1.0 will favour laser procedure. The parameters with asterisk are statistically significant 
points. In all patients the shrinkage of the obliterated vein (expressed as the mean percentage change of the vein's diameter) was most apparent at the 5 pter position and was most pronounced six months after the procedure (Fig. 3). Of note, the mean diameters as well as the mean diameter changes in respective control points between the VB and L groups did not differ statistically.

The assessment of procedure efficacy performed after one month revealed complete occlusion without any blood flow in all treated veins in the L group (100\%). In contrast, two out of 41 veins $(4.9 \%)$ in the VB group were only partially occluded. After six months (Fig. 4) partial recanalisation was noted in one of the treated veins from the L group (2.2\%), whereas an additional two veins in the VB group $(9.7 \%)$ became partially recanalised (Fig. 5). The observed difference between both groups after six months, although noticeable, was not statistically significant $(p=0.07)$. The OR assessed for vein recanalisation within six months after the procedure in the VB group was 4.86 with $95 \% \mathrm{CI}=0.52-45.42$ and $p=0.16$ (Fig. 2). Of note, the mean initial diameters at the ter or pter levels of recanalised veins in both groups were above the mean values for each group $(8.8 \pm 0.9 \mathrm{~mm}$ vs. $7.3 \pm 1.8 \mathrm{~mm}$ in the VB group and $7.6 \pm 1.5 \mathrm{~mm}$ in the L group, respectively).

\section{DISCUSSION}

Modern treatment of truncal venous insufficiency should follow patients' and their doctors' expectations. The ideal procedure should be minimally invasive, fast, painless (or with only reasonable discomfort), and effective. Preferably, it should be applicable in ambulatory settings, optimally without any sophisticated equipment.

The endovenous procedure with cyanoacrylate glue, the VenaBlock system, seems to meet at least most of the aforementioned expectations. It does not require hospital stay and can be performed in ambulatory settings. Except for single cases, it is almost painless and requires local anaesthesia only in the place of catheter introduction. Because there is no need for use of time-consuming tumescent anaesthesia, the duration of the VenaBlock procedure depends mainly on the time required for vein access and precise placement of the catheter tip. This property of a glue-based method significantly reduces the time of staff engagement and operating room occupation in comparison with endovenous laser systems. In our study, the application of glue required almost one-third of the time needed for a typical procedure with the use of thermal ablation.

On the other hand, lack of tumescent anaesthesia, although indicated as one of main benefits of endovenous glue-based systems, may result in some disadvantages for the patient. Obviously, due to many punctures and discomfort associated with application of Klein's solution around the treated vein, the use of tumescence was initially recognised by some patients as uncomfortable. However, later stages of the procedure were completely painless. Moreover, the tumescence empties the vein and prevents blood inflow, thus decreasing the risk of residual thrombus-related phlebitis. Therefore, the use of tumescence in the L group, in comparison with the VB group, was associated with reduced pain and less discomfort within seven days after the procedure. Pain was seen more frequently in patients from the VB group and resulted mainly from mild inflammatory reaction along treated veins, especially those of larger diameter. Presumably, the difference in the level of postoperative pain could also be associated with different post-procedural recommendations regarding wearing of compression stockings [7]. Similarly to tumescence, no use of compression stockings after VenaBlock procedure, although suggested as more comfortable for the patient, could paradoxically result in increased frequency of adverse reactions. This conclusion may be supported by the observed lower intensity or lack of inflammatory symptoms in patients who used compression after glue-based procedures (unpublished data).

A comparison of the efficacies of both methods seems to be the most interesting. Of note, there were no significant differences between the groups before the treatment. Furthermore, all procedures were performed by the same team who were experienced in the ELVeS and VenaBlock systems. Therefore, it is plausible that the effectiveness of each method, observed in our study, resulted directly from the unique properties of both systems and was not biased by any other modifying factor.

The ELVeS procedure involved application of tumescence, slow and precise vein heating, and, finally, the use of compression stockings during the post-operative period. By contrast, in the VenaBlock system catheter traction is very fast, and therefore it may not guarantee uniform glue application and shrinkage/ occlusion of a large vein. This condition may be critical, especially in the presence of blood persisting in the vein's lumen or an inflow from tributaries shortly after the procedure. The presence of blood inside the vein may result in formation of small thrombi within some segments of occluded vessels, with subsequent fibrinolysis and recanalisation. This assumption could be supported by a higher frequency of vein recanalisation in the VB group, especially in patients presenting with larger veins. In fact, our data suggest that the clinical efficacy of the VenaBlock system was at least comparable to laser thermoablation in small- and medium-diameter truncal vein incompetence. However, contrary to some other reports [6], efficacy was inferior to ELVeS if larger veins were managed. In comparison with ELVeS, the VenaBlock is much faster and more cost-effective because it does not require any additional equipment except for a standard sonographic set. Moreover, due to the lower number of punctures needed to deliver local 

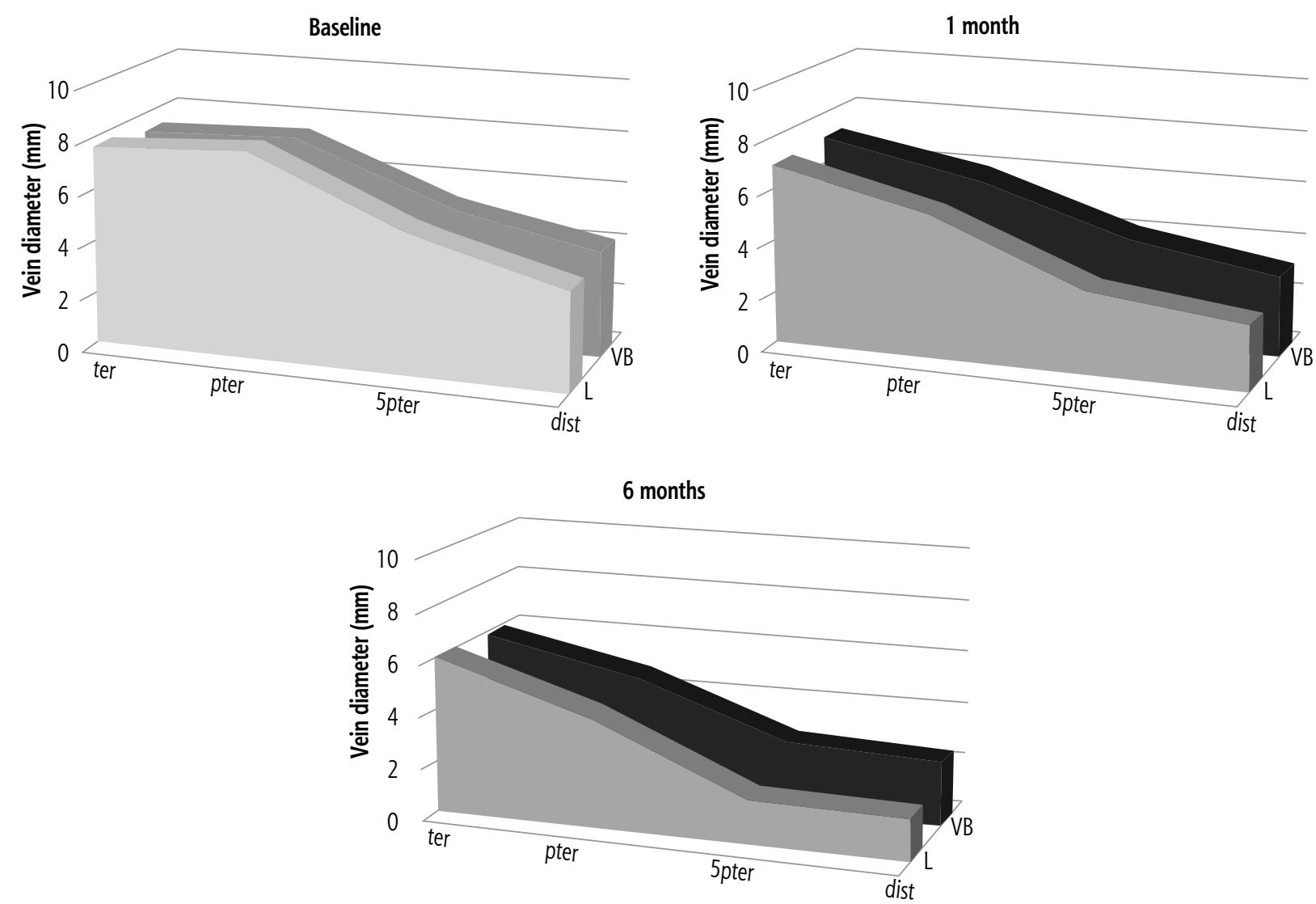

Fig. 3. Comparison of mean vein diameters at different levels between both groups - at baseline and after 1 month and 6 months after procedure (VB - VenaBlock group, L - laser group, ter - terminal valve level, pter - pre-terminal valve level, $5 \mathrm{pter}-5 \mathrm{~cm}$ below pre-terminal valve level, dist - distal reflux point level)
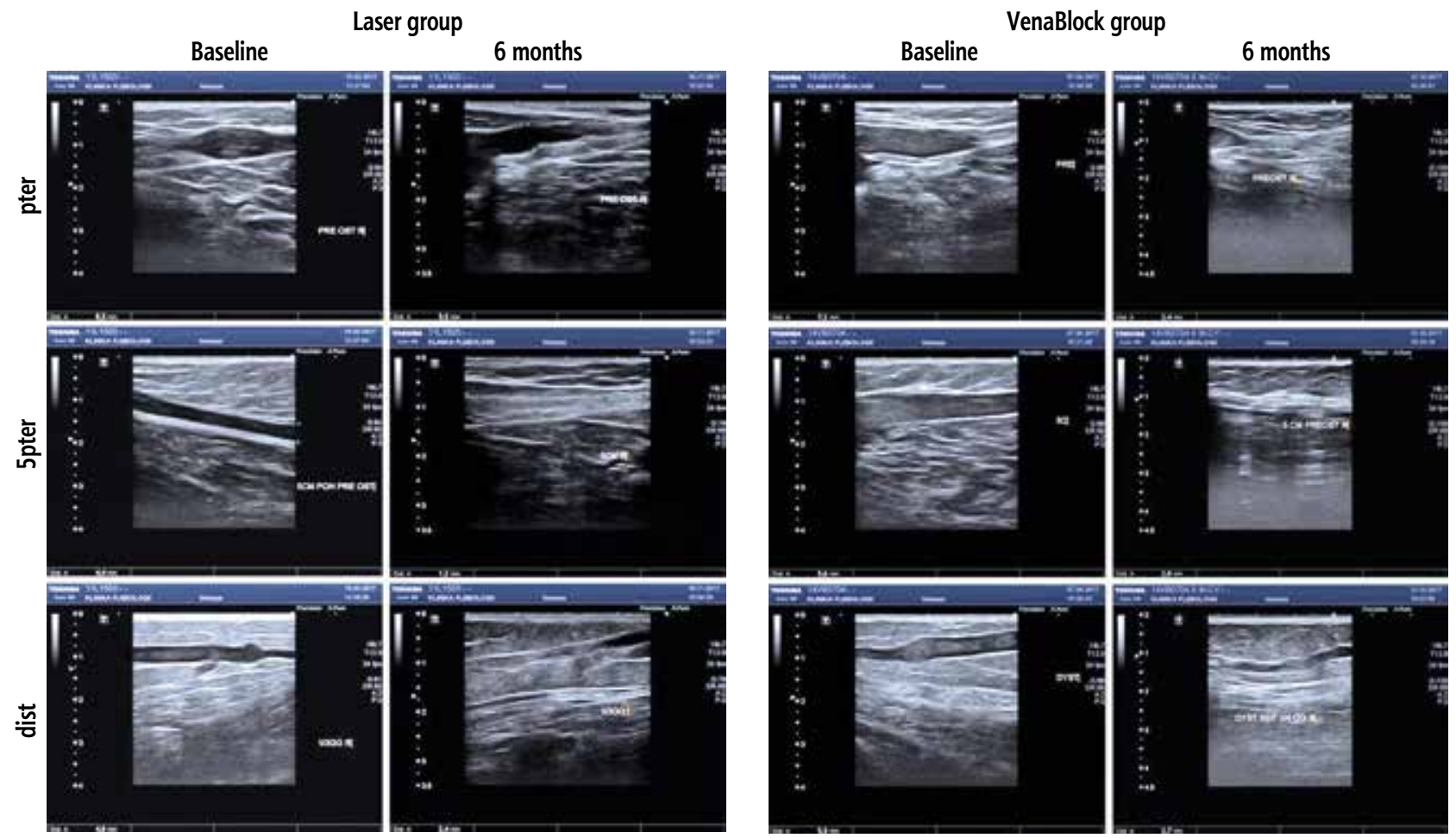

Fig. 4. Ultrasound scan sets of representative patients from both groups at baseline and 6 months after procedure (pter - pre-terminal valve level, 5 pter $-5 \mathrm{~cm}$ below pre-terminal valve level, dist - distal reflux point level) 
anaesthesia and no compression after the procedure, patients may prefer the glue method over laser thermoablation with tumescent anaesthesia.

\section{CONCLUSIONS}

The VenaBlock glue system is a safe, effective, and extremely quick procedure. The minimal dose of local anaesthesia and no need for compression after the procedure makes this system an attractive alternative to infrared laser thermoablation. On the other hand, glue system appears to be less versatile than ELVeS, especially for the treatment of larger diameter veins. Due to slightly lower efficacy in comparison with ELVeS, the use of the VenaBlock seems to be limited to small- and medium-diameter veins. Its use in large-diameter veins was more frequently accompanied by local inflammation along the treated vessel and noticeable risk of its recanalisation (although in our experience that disadvantage could easily be overcome by application of tumescence and/ or post-procedural compression). However, this issue requires further studies.

\section{ACKNOWLEDGEMENT}

A. Krawczyk was involved in preparation of documents for Ethical Board approval and in patients recruitment.

The authors declare no conflict of interest.

\section{References}

1. Lawaetz M, Serup J, Lawaetz B, et al. Comparison of endovenous ablation techniques, foam sclerotherapy and surgical stripping for great saphenous varicose veins. Extended 5-year follow-up of a RCT. Int Angiol 2017; 36: 281-288.

2. Vos CG, Ünlü Ç, Bosma J, et al. A systematic review and meta-analysis of two novel techniques of nonthermal endovenous ablation of the great saphenous vein. J Vasc Surg Venous Lymphat Disord 2017; 5: 880-896.

3. Almeida JI, Javier JJ, Mackay E, et al. First human use of cyanoacrylate adhesive for treatment of saphenous vein incompetence. J Vasc Surg Venous Lymphat Disord 2013; 1: 174-180.

4. Yasim A, Eroglu E, Bozoglan O, et al. A new non-tumescent endovenous ablation method for varicose vein treatment: Early results of N-butyl cyanoacrylate (VariClose). Phlebology 2017; 32: 194-199.

5. Morrison N, Gibson K, Vasquez M, et al. VeClose trial 12-month outcomes of cyanoacrylate closure versus radiofrequency ablation for incompetent great saphenous veins. J Vasc Surg Venous Lymphat Disord 2017; 5: 321-330.
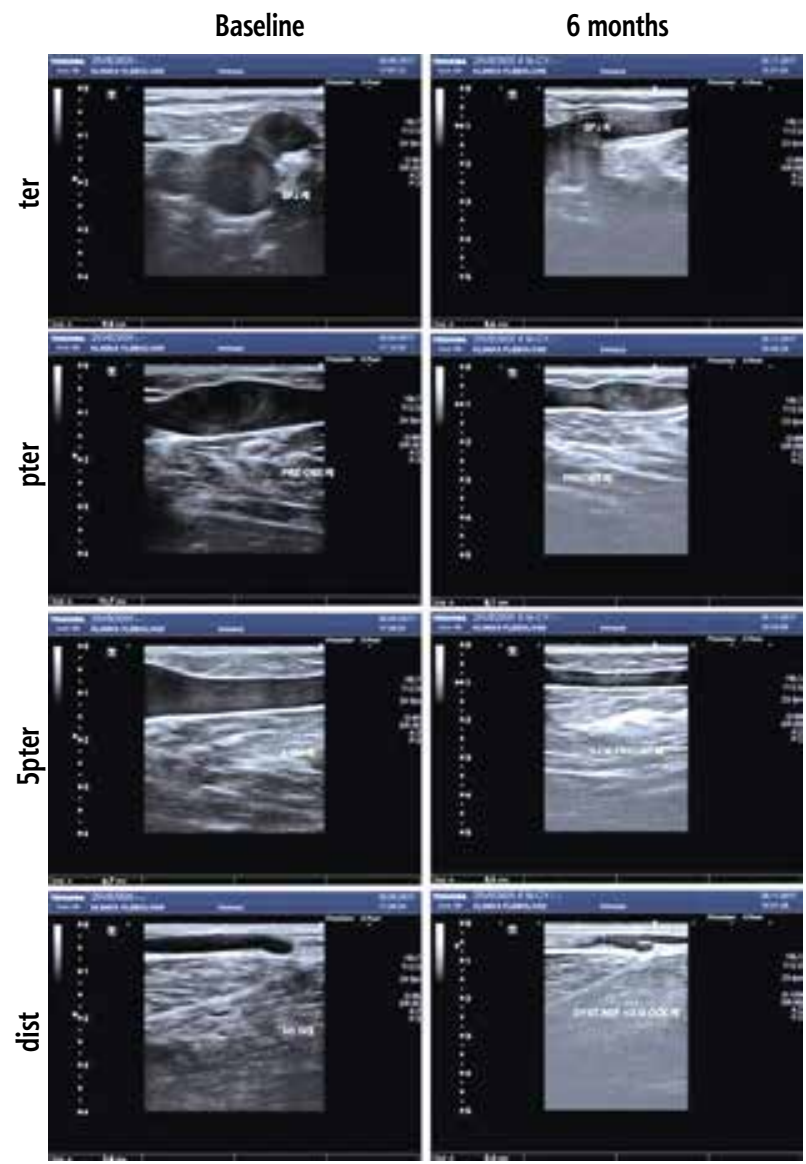

Fig. 5. Ultrasound scan set of a patient from the VenaBlock group with almost complete recanalisation within 6 months after the procedure. The only short fragment of treated vein near the distal reflux point remained occluded (ter - terminal valve level, pter - pre-terminal valve level, $5 \mathrm{pter}-5 \mathrm{~cm}$ below pre-terminal valve level, dist - distal reflux point level)

6. Bozkurt AK, Yllmaz MF. A prospective comparison of a new cyanoacrylate glue and laser ablation for the treatment of venous insufficiency. Phlebology 2016; 31 (Suppl): 106-113.

7. Gibson K, Ferris B. Cyanoacrylate closure of incompetent great, small and accessory saphenous veins without the use of post-procedure compression: Initial outcomes of a post-market evaluation of the VenaSeal System (the WAVES Study). Vascular 2017; 25: 149-156.

8. Klein JA. The tumescent technique for liposuction surgery. Am J Cosmetic Surg 1987; 4: 1124-1132. 